\title{
MEAN-FIELD- AND CLASSICAL LIMIT OF MANY-BODY SCHRÖDINGER DYNAMICS FOR BOSONS
}

\author{
Jürg Fröhlich ${ }^{1}$, Sandro Graffi ${ }^{2}$, Simon Schwarz ${ }^{3}$
}

\begin{abstract}
We present a new proof of the convergence of the $N$-particle Schrödinger dynamics for bosons towards the dynamics generated by the Hartree equation in the mean-field limit. For a restricted class of two-body interactions, we obtain convergence estimates uniform in $\hbar$, up to an exponentially small remainder. For $\hbar=0$, the classical dynamics in the mean-field limit is given by the Vlasov equation.
\end{abstract}

\section{Introduction and statement of results}

Consider the Schrödinger operator

$$
\begin{aligned}
& H_{N}=H_{N}^{0}+W_{N} \\
& H_{N}^{0}=-\sum_{i=1}^{N} \frac{\hbar^{2}}{2} \Delta_{i}, \quad W_{N}=\frac{1}{N} \sum_{i<j}^{N} w\left(x_{i}-x_{j}\right)
\end{aligned}
$$

where $w$ is a two-body potential independent of $N$. The operator $H_{N}$ acts on $\mathcal{H}^{(N)}:=$ $L_{S}^{2}\left(\mathbb{R}^{3 N}\right)$, the totally symmetric part of $L^{2}\left(\mathbb{R}^{3 N}\right)$, which is the Hilbert space of pure state vectors for a system of $N$ nonrelativistic bosons. We propose to study the dynamics described by the $N$-body Schrödinger equation

$$
i \hbar \partial_{t} \Psi_{N}(t)=H_{N} \Psi_{N}(t)
$$

for an inital condition $\Psi_{N}(t=0)=\Psi_{N, 0} \in L_{S}^{2}\left(\mathbb{R}^{3 N}\right)$. Under assumptions specified below, $H_{N}$, defined on the symmetrized Sobolev space $H_{S}^{2}\left(\mathbb{R}^{3 N}\right)$, is a self-adjoint operator. Hence the unitary group $U_{N}(t)=e^{-i H_{N} t / \hbar}, t \in \mathbb{R}$, exists. Let $p \leq N$, and let $a^{(p)}$ be a bounded operator on $L_{S}^{2}\left(\mathbb{R}^{3 p}\right)$. It defines an operator $A_{N}^{(p)}$ acting on $\mathcal{H}^{(N)}$ in the following way:

$$
\begin{aligned}
& \left(A_{N}^{(p)} \Psi\right)\left(x_{1}, \ldots, x_{N}\right)=\frac{N(N-1) \cdots(N-p+1)}{N^{p}}\left(P_{S} a^{(p)} \otimes I^{(N-p)} P_{S} \Psi\right)\left(x_{1}, \ldots, x_{N}\right), \\
& \Psi\left(x_{1}, \ldots, x_{N}\right) \in L_{S}^{2}\left(\mathbb{R}^{3 N}\right),
\end{aligned}
$$

where $P_{S}$ is the projection onto the symmetric subspace $L_{S}^{2}\left(\mathbb{R}^{3 N}\right)$ of $L^{2}\left(\mathbb{R}^{3 N}\right)$. The operator $A_{N}^{(p)}$ may be viewed as an operator acting on $p$ particles; the numerator on the right side

\footnotetext{
${ }^{1}$ Theoretische Physik, ETH Zürich, Switzerland. (juerg@itp.phys.ethz.ch)

${ }^{2}$ Dipartimento di Matematica, Università di Bologna, Italy. (graffi@dm.unibo.it)

${ }^{3}$ Theoretische Physik, ETH Zürich, Switzerland. (sschwarz@itp.phys.ethz.ch)
} 
of (1.4) is a combinatorial factor motivated by "second quantization"; the denominator is the correct scaling factor to take the $N \rightarrow \infty$ limit.

We are interested in the asymptotics of certain expectation values of the Heisenbergpicture operators $e^{i H_{N} t / \hbar} A_{N}^{(p)} e^{-i H_{N} t / \hbar}$, as $N \rightarrow \infty$. If $H_{N}$ is chosen as in (1.1), (1.2), and $A_{N}^{(p)}$ is chosen as in (1.4), the limit $N \rightarrow \infty$ is the usual mean-field limit; see [He, Sp].

Our first main result is the following

Theorem 1.1. Let $\hbar>0$ and $t \geq 0$ be fixed, and let $w \in L^{\infty}\left(\mathbb{R}^{3}\right)$. If $\Psi_{N, 0}\left(x_{1}, \ldots, x_{N}\right)=$ $\psi\left(x_{1}\right) \cdots \psi\left(x_{N}\right)$ is a normalized "coherent" (i.e., product) initial state, then

$$
\begin{gathered}
\lim _{N \rightarrow \infty}\left\langle\Psi_{N, 0}, e^{i H_{N} t / \hbar} A_{N}^{(p)} e^{-i H_{N} t / \hbar} \Psi_{N, 0}\right\rangle= \\
\lim _{N \rightarrow \infty}\left\langle\Psi_{N, t}, A_{N}^{(p)} \Psi_{N, t}\right\rangle=\left\langle\Psi_{p, t}, a^{(p)} \Psi_{p, t}\right\rangle=: a^{(p)}\left(\psi_{t}\right)
\end{gathered}
$$

Here $\Psi_{N, t}$ is again a coherent state, i.e., $\Psi_{N, t}\left(x_{1}, \ldots, x_{N}\right)=\psi_{t}\left(x_{1}\right) \cdots \psi_{t}\left(x_{N}\right)$, and $\Psi_{p, t}=$ $\Psi_{N=p, t}$, where $\psi_{t}$ is a solution of the Hartree equation

$$
i \hbar \partial_{t} \psi_{t}=-\frac{\hbar^{2}}{2} \Delta \psi_{t}+\left(w *\left|\psi_{t}\right|^{2}\right) \psi_{t}
$$

with initial condition $\psi_{t=0}=\psi$.

\section{Remarks}

1. For large $N$, the quantum evolution $e^{-i H_{N} t / \hbar} \Psi_{N, 0}$ can be replaced by the nonlinear single-particle evolution $\Psi_{N, t}\left(x_{1}, \ldots, x_{N}\right)$. Particle interaction effects are translated into the nonlinearity of this evolution. This justifies interpreting the limit $N \rightarrow \infty$ as a mean-field limit.

2. The corrections to the limit in $(1.5)$ are $O(1 / N)$.

3. Since $\lim _{N \rightarrow \infty} N(N-1) \cdots(N-p+1) / N^{p}=1$, the second equality in (1.5) follows easily from (1.4), because

$$
\lim _{N \rightarrow \infty}\left\langle\Psi_{N, t}, A_{N}^{(p)} \Psi_{N, t}\right\rangle=\left\langle\Psi_{p, t}, a^{(p)} \Psi_{p, t}\right\rangle_{L^{2}\left(\mathbb{R}^{3 p}\right)}\|\psi\|_{L^{2}\left(\mathbb{R}^{3}\right)}^{2(N-p)}
$$

4. Theorem 1.1 was first proven in [He], see also [GiVe]. A new proof was given in [Sp] and extended to more general classes of two-body potentials, including the Coulomb potential, in $[\mathrm{EY}],[\mathrm{BGM}],[\mathrm{BEGMY}]$. The proof in our paper is quite different. It is inspired by a second-quantization formalism to be published elsewhere. It enables us to tackle the problem of obtaining convergence estimates uniform in Planck's constant $\hbar$, as we now proceed to discuss. 
It is well known that, for $W_{N}$ as in (1.2), the classical dynamics of $N$ particles tends to the dynamics defined by the Vlasov equation, in the limit $N \rightarrow \infty$. More precisely, if $\rho_{N}$ denotes the empirical distribution, namely

$$
\rho_{N}(d x, d \xi ; t)=\frac{1}{N} \sum_{i=1}^{N} \delta\left(x-x_{i}(t)\right) \delta\left(\xi-\xi_{i}(t)\right) d x d \xi
$$

where $\left(x_{1}(t), \ldots, x_{N}(t) ; \xi_{1}(t), \ldots, \xi_{N}(t)\right)$ is a solution of the classical equations of motion, then, in the limit $N \rightarrow \infty, \rho_{N}$ tends weakly to $f_{t}(x, \xi) d x d \xi$, where $f_{t}(x, \xi)$ is a solution of the Vlasov equation:

$$
\begin{aligned}
\partial_{t} f_{t} & =-\xi \cdot \nabla_{x} f_{t}+\nabla_{x} V_{e f f} \cdot \nabla_{\xi} f_{t} \\
V_{e f f}(x, t) & =\int w(x-y) f_{t}(y, \xi) d y d \xi
\end{aligned}
$$

see $[\mathrm{BH}]$. It is natural to ask whether this convergence result is related to that of Theorem 1.1. Our next result provides, under very restrictive assumptions on the two-body interactions, a partial answer to this question. First, we define a restricted class of interactions. For $\sigma>0$, we define the spaces

$$
\begin{aligned}
& L_{\sigma, p}^{1}:=\left\{f \in L^{1}\left(\mathbb{R}^{6 p}\right) \mid e^{\sigma|z|} f \in L^{1}\left(\mathbb{R}^{6 p}\right)\right\} \\
& \mathcal{A}_{\sigma, p}:=\left\{f \in L^{1}\left(\mathbb{R}^{6 p}\right) \mid e^{\sigma|s|} \widehat{f} \in L^{1}\left(\mathbb{R}^{6 p}\right)\right\}
\end{aligned}
$$

Here $x_{j} \in \mathbb{R}^{3}, \xi_{j} \in \mathbb{R}^{3}, j=1, \ldots, p$, and

$$
\begin{gathered}
z:=\left(X_{p}, \Xi_{p}\right) \in \mathbb{R}^{3 p} \times \mathbb{R}^{3 p} ; X_{p}:=\left(x_{1}, \ldots, x_{p}\right), \Xi_{p}:=\left(\xi_{1}, \ldots, \xi_{p}\right), \\
|z|:=\sum_{j=1}^{p}\left(\left|x_{j}\right|+\left|\xi_{j}\right|\right) ;
\end{gathered}
$$

$\widehat{f}(s), s:=(S, \Sigma) \in \mathbb{R}^{3 p} \times \mathbb{R}^{3 p}$ is the Fourier transform of $f$.

We further denote by $\Phi_{t}^{N}:\left(X_{N} ; \Xi_{N}\right) \mapsto\left(X_{N}(t) ; \Xi_{N}(t)\right)$ the flow generated by $H_{N}^{c}$, where $H_{N}^{c}$ is the classical Hamilton function corresponding to the operator $H_{N}$.

Definition 1.1 We define by:

1.

$$
\begin{aligned}
& W_{N}^{\Psi_{N}}\left(X_{N}, \Xi_{N} ; t\right)= \\
& (2 \pi)^{-3 N} \int_{\mathbb{R}^{3 N}} e^{i\left\langle Y_{N}, \Xi_{N}\right\rangle} \Psi_{N}\left(X_{N}+\hbar Y_{N} / 2, t\right) \bar{\Psi}_{N}\left(X_{N}-\hbar Y_{N} / 2, t\right) d Y_{N}
\end{aligned}
$$

the Wigner distribution of the $N$-particle normalized wave function $\Psi_{N}\left(X_{N}, t\right)$; 
2.

$$
W_{j}^{\Psi_{N}}\left(X_{j}, \Xi_{j} ; t\right)=\int_{\mathbb{R}^{3(N-j)}} W_{N}^{\Psi_{N}}\left(X_{N}, \Xi_{N} ; t\right) d X_{N-j} d \Xi_{N-j},
$$

the $j$-particle Wigner function $((N-j)$-marginal distribution of the $N$-particle Wigner distribution).

3.

$$
W(\psi)(x, \xi ; t)=(2 \pi)^{-3} \int_{\mathbb{R}^{3}} e^{i\langle y, \xi\rangle} \psi_{t}(x+\hbar y / 2) \overline{\psi_{t}}(x-\hbar y / 2) d y,
$$

the Wigner distribution of the solution $\psi_{t}(x)$ of the Hartree equation.

Our second main result is

Theorem 1.2 . Let $w \in \mathcal{A}_{\sigma, 1}$, for some $\sigma>0$. Let $\Psi_{N, 0}$ be a product state. Set $\epsilon:=$ $\|w\|_{\infty} t$. Then, for fixed $p$, there is a constant $C_{p}>0$ independent of $\hbar$ such that, as an equality between tempered distributions,

$$
W_{p}^{\Psi_{N}}\left(X_{p}, \Xi_{p} ; t\right)=\prod_{j=1}^{p} W(\psi)\left(x_{j}, \xi_{j} ; t\right)+\frac{C_{p}}{N}+O\left(e^{-1 / \sqrt{\epsilon}}\right),
$$

as $N \rightarrow \infty$.

\section{Remarks}

1. It is known that $W(\psi)(x, \xi ; t)$ converges in $\mathcal{S}^{\prime}\left(\mathbb{R}^{6}\right)$ to a solution $f_{t}(x, \xi)$ of the Vlasov equation, as $\hbar \rightarrow 0$ [NS]. It is also known that

$$
\lim _{N \rightarrow \infty} W_{p}^{\Psi_{N}}\left(X_{p}, \Xi_{p} ; t\right)=\prod_{j=1}^{p} f_{t}\left(x_{j}, \xi_{j}\right)
$$

whenever $N \rightarrow \infty$ entails $\hbar \rightarrow 0$, as in the case of the Kac potentials [NS],[GMP].

2. Result (1.14) shows that, up to an exponentially small error independent of $\hbar$, the mean-field convergence towards a single-particle nonlinear dynamics holds uniformly in $\hbar$.

3. The classical limit is equivalent to the limit of heavy particles. We set $\hbar=1$ in (1.2), but let the particle mass $m$ become large. We impose the condition that the kinetic energy per particle be independent of $m$, namely $m v_{i}^{2}=O(1)$, i.e., $\left|v_{i}\right|=O(1 / \sqrt{m})$, for all $i$. This suggests to rescale time as $t=\sqrt{m} \tau$. Then the Schrödinger equation becomes

$$
\frac{i}{\sqrt{m}} \partial_{\tau} \Psi_{N}=\sum_{j=1}^{N}-\frac{\Delta_{j}}{2 m} \Psi_{N}+\frac{1}{N} \sum_{i, j=1}^{N} w\left(x_{i}-x_{j}\right) \Psi_{N},
$$

which is equivalent to (1.1)-(1.3), for $\hbar=1 / \sqrt{m}$. 


\section{The $N \rightarrow \infty$ limit: convergence estimates}

\subsection{Kinematical algebra of "observables"}

The above systems can be described by a kinematical algebra of operators, the quantum mechanical analogue of the algebra of functions on phase space of a classical system.

Let $\mathcal{H}^{(p)}:=L_{S}^{2}\left(\mathbb{R}^{3 p}\right), 0<p<N, N \in \mathbb{N}$. Let $a^{(p)}$ be a bounded operator on $\mathcal{H}^{(p)}$, and $\alpha^{(p)}\left(x_{1}, \ldots, x_{p} ; y_{1}, \ldots, y_{p}\right):=\alpha^{(p)}\left(X_{p} ; Y_{p}\right)$ be the tempered distribution kernel in $\mathcal{S}^{\prime}\left(\mathbb{R}^{3 p} \times\right.$ $\left.\mathbb{R}^{3 p}\right)$ associated to $a^{(p)}$ by the nuclear theorem:

$$
\left(a^{(p)} \varphi^{(p)}\right)\left(X_{p}\right)=\int_{\mathbb{R}^{p}} \alpha^{(p)}\left(X_{p} ; Y_{p}\right) \varphi^{(p)}\left(Y_{p}\right) d Y_{p}
$$

where $\varphi^{(p)}\left(Y_{p}\right) \in L_{S}^{2}\left(\mathbb{R}^{3 p}\right)$. Then $\left(a^{(p)}\right)^{*}$ has the distribution kernel $\overline{\alpha^{(p)}\left(Y_{p} ; X_{p}\right)}$.

To $a^{(p)}$ we associate the operator $A_{N}^{(p)}$ on $L_{S}^{2}\left(\mathbb{R}^{3 N}\right)$ specified in (1.4). Explicitly:

$$
A_{N}^{(p)}\left(a^{(p)}\right) \varphi^{(N)}:=\left(\begin{array}{c}
N \\
p
\end{array}\right) \frac{p !}{N^{p}} P_{S} \int_{\mathbb{R}^{3 N}} K\left((\cdot), Y_{N}\right) \varphi^{(N)}\left(Y_{N}\right) d Y_{N},
$$

with

$$
K\left(X_{N}, Y_{N}\right)=\alpha^{(p)}\left(X_{p} ; Y_{p}\right) \delta\left(X_{N-p}-Y_{N-p}\right), \varphi^{(N)}\left(Y_{N}\right) \in L_{S}^{2}\left(\mathbb{R}^{3 N}\right)
$$

If $a^{(p)}$ is bounded on $\mathcal{H}^{(p)}$ then $A_{N}^{(p)}\left(a^{(p)}\right)$ is bounded on $\mathcal{H}^{(N)}$. Since $\left\|P_{S}\right\|=1$ and $\left(\begin{array}{c}N \\ p\end{array}\right) \frac{p !}{N^{p}} \leq 1$, we have that

$$
\begin{aligned}
& \left\|A_{N}^{(p)} \varphi^{(N)}\right\|_{\mathcal{H}^{(N)}}^{2} \leq\left\|a^{(p)}\right\|_{\mathcal{H}^{(p)}}^{2} \int_{R^{3(N-p)}}\left(\int_{R^{3 p}}\left|\varphi^{(N)}\left(Y_{p} ; X_{N-p}\right)\right|^{2} d Y_{p}\right) d X_{N-p} \\
& =\left\|a^{(p)}\right\|_{\mathcal{H}^{(p)}}^{2}\left\|\varphi^{(N)}\right\|_{\mathcal{H}^{(N)}}^{2}
\end{aligned}
$$

We set

$$
\widehat{\mathcal{A}}_{N}:=\left\langle A_{N}^{(p)}\left(a^{(p)}\right) \mid a^{(p)} \in B\left(\mathcal{H}^{(p)}\right), p=0,1,2, \ldots\right\rangle \subset B\left(\mathcal{H}^{(N)}\right)
$$

The following statement is easily verified.

Proposition 2.1. The map $a^{(p)} \mapsto A_{N}^{(p)}\left(a^{(p)}\right)$ is linear, and $\left(A_{N}^{(p)}\left(a^{(p)}\right)\right)^{*}=A_{N}^{(p)}\left(\left(a^{(p)}\right)^{*}\right)$, $\left\|A_{N}^{(p)}\left(a^{(p)}\right)\right\|_{B\left(\mathcal{H}^{(N)}\right)} \leq\left\|a^{(p)}\right\|_{B\left(\mathcal{H}^{(p)}\right)}$.

\subsection{The Schwinger-Dyson expansion}

Given a bounded operator $A_{N}^{(p)}$ acting on $\mathcal{H}^{(N)}, p \leq N$, we denote by $A_{t, N}^{(p)}$ the corresponding Heisenberg-picture operator with respect to the free time evolution $e^{i H_{N}^{0} t / \hbar}$, i.e.,

$$
A_{t, N}^{(p)}=e^{i H_{N}^{0} t / \hbar} A_{N}^{(p)} e^{-i H_{N}^{0} t / \hbar}
$$


We further denote by $A_{N}^{(p)}(t)$ the corresponding operator with $H_{N}^{0}$ replaced by $H_{N}$, namely

$$
A_{N}^{(p)}(t):=e^{i H_{N} t / \hbar} A_{N}^{(p)} e^{-i H_{N} t / \hbar}
$$

and by $A_{I, N}^{(p)}(t, s)$ the two-parameter operator family

$$
A_{I, N}^{(p)}(t, s):=e^{i H_{N} t / \hbar} e^{-i H_{N}^{0} t / \hbar} A_{s, N}^{(p)} e^{i H_{N}^{0} t / \hbar} e^{-i H_{N} t / \hbar}
$$

Then we obviously have

$$
A_{N}^{(p)}(t)=\left.A_{I, N}^{(p)}(t, s)\right|_{s=t}
$$

We denote $W_{N}$ by $W$. Iterating the identity:

$$
A_{I, N}^{(p)}(t, s)=A_{s, N}^{(p)}+\frac{i}{\hbar} \int_{0}^{t} e^{i H_{N} t_{1} / \hbar} e^{-i H_{N}^{0} t_{1} / \hbar}\left[W_{t_{1}}, A_{s, N}^{(p)}\right] e^{i H_{N}^{0} t / \hbar} e^{-i H_{N} / \hbar} d t_{1}
$$

we get that

$$
A_{I, N}^{(p)}(t, s)=A_{s, N}^{(p)}+\sum_{n=1}^{\infty}\left(\frac{i}{\hbar}\right)^{n} \int_{0}^{t} d t_{1} \ldots \int_{0}^{t_{n-1}} d t_{n}\left[W_{t_{n}}, \ldots,\left[W_{t_{1}}, A_{s, N}^{(p)}\right] \ldots\right]
$$

and finally, setting $s=t$, we obtain the Schwinger-Dyson expansion

$$
\left.A_{N}^{(p)}(t)=A_{t, N}^{(p)}+\sum_{n=1}^{\infty}\left(\frac{i}{\hbar}\right)\right)^{n} \int_{0}^{t} \int_{0}^{t_{1}} \ldots \int_{0}^{t_{n-1}}\left[W_{t_{n}}, \ldots,\left[W_{t_{1}}, A_{t, N}^{(p)}\right] \ldots\right] d t_{n} \ldots d t_{1}
$$

From now on, we drop the index $N$ in the Heisenberg-picture operators with respect to the free evolution, i.e. we use the abbreviation: $A_{t, N}^{(p)}:=A_{t}^{(p)}$.

The boundedness of $A_{N}^{(p)}$ and of the interactions $W_{t_{i}}$ implies the boundedness of all multiple commutators, with

$$
\begin{aligned}
\frac{1}{\hbar^{n}}\left\|\left[W_{t_{n}}, \ldots,\left[W_{t_{1}}, A_{t}^{(p)}\right] \ldots\right]\right\|_{\mathcal{H}^{(N)}} & \leq\left(2\|W\|_{\mathcal{H}^{(N)}} / \hbar\right)^{n}\left\|A_{N}^{(p)}\right\|_{\mathcal{H}^{(N)}} \\
& \leq\left(2\|W\|_{\mathcal{H}^{(N)}} / \hbar\right)^{n}\left\|a^{(p)}\right\|_{\mathcal{H}^{(p)}}
\end{aligned}
$$

for $A_{N}^{(p)}=A_{N}^{(p)}\left(a^{(p)}\right)$. By (1.2), $\|W\|_{\mathcal{H}^{(N)}} \propto N$. Hence, for fixed $N$ and $\hbar$, the series is norm-convergent, for all $t \geq 0$. The time integrations yield a factor $\frac{t^{n}}{n !}$, so that the norm of the series in (2.11) is bounded by $\exp \left[2\|W\|_{\mathcal{H}^{(N)}}|t| / \hbar\right] \cdot\left\|a^{(p)}\right\|_{\mathcal{H}^{(p)}}$.

These estimates are obviously not adequate to investigate the $N \rightarrow \infty$ or the $\hbar \rightarrow 0$ limit, let alone to prove uniformity in $\hbar$. 


\subsection{The $N \rightarrow \infty$ limit}

We exploit the structure of the commutators on the right-hand side of (2.11), the symmetry of wave functions in $L_{S}^{2}\left(\mathbb{R}^{3 N}\right)$, and the fact that each term in $A_{N}^{(p)}$ only acts on $p$ arguments of a wave function, so that many commutators will vanish. Note that

$$
W_{t}=\frac{1}{N} \sum_{i<j}^{N} e^{-i H_{N}^{0} t / \hbar} w^{i j} e^{i H_{N}^{0} t / \hbar}=\frac{1}{N} \sum_{i<j}^{N} w_{t}^{i j}
$$

where

$$
w_{t}^{i j}=e^{i\left(\Delta_{i}+\Delta_{j}\right) t \hbar / 2} w^{i j} e^{-i\left(\Delta_{i}+\Delta_{j}\right) t \hbar / 2}, w^{i j}=w\left(x_{i}-x_{j}\right) .
$$

Therefore

$$
\begin{aligned}
{\left[W_{s}, A_{N}^{(p)}\right] } & =\frac{1}{N} \sum_{i<j}^{N}\left[w_{s}^{i j}, A_{N}^{(p)}\right]= \\
& =\frac{1}{N} \sum_{i=1}^{p} \sum_{j=p+1}^{N} A_{N}^{(p+1)}\left(\left[w_{s}^{i j}, a^{(p)}\right]\right)+\frac{1}{N} \sum_{i<j}^{p} A_{N}^{(p)}\left(\left[w_{s}^{i j}, a^{(p)}\right]\right)= \\
& =\frac{N-p}{N} \sum_{i=1}^{p} A_{N}^{(p+1)}\left(\left[w_{s}^{i p+1}, a^{(p)}\right]\right)+\frac{1}{N} \sum_{i<j}^{p} A_{N}^{(p)}\left(\left[w_{s}^{i j}, a^{(p)}\right]\right)
\end{aligned}
$$

In more precise terms, the expression

$$
\left[W_{s}, A_{N}^{(p)}\right]=\frac{N-p}{N} \sum_{i=1}^{p} A_{N}^{(p+1)}\left(\left[w_{s}^{i p+1}, a^{(p)}\right]\right)+\frac{1}{N} \sum_{i<j}^{p} A_{N}^{(p)}\left(\left[w_{s}^{i j}, a^{(p)}\right]\right),
$$

holds as an operator identity on $\mathcal{H}^{(N)}$. In second-quantization language, the first sum on (2.15) corresponds to tree graphs, the second one to loop graphs. Next, we insert (2.15) in (2.9) and perform a second step, but only for the first sum in (2.15), leaving the second one unchanged. To keep our notation compact, it is useful to introduce the notion of tree amplitudes of $n$-th order, recursively defined in the following way

$$
g^{(0, p)}=a^{(p)} ; \quad g_{t_{1}, \ldots, t_{n}}^{(n ; p)}=\frac{i}{\hbar} \sum_{i=1}^{p+n-1}\left[w_{t_{n}}^{i p+n}, g_{t_{1}, \ldots, t_{n-1}}^{(n-1 ; p)}\right], \quad n \geq 1
$$

Then expression (2.15) becomes

$$
\frac{i}{\hbar}\left[W_{s}, A_{N}^{(p)}\right]=\frac{N-p}{N} A_{N}^{(p+1)}\left(g_{s}^{(1 ; p)}\right)+\frac{i}{N \hbar} \sum_{i<j}^{p} A_{N}^{(p)}\left(\left[w_{s}^{i j}, g^{(0 ; p)}\right]\right) .
$$

The first term is $O(1)$, while the second one is of order $p(p-1) / N$ (for fixed $\hbar$ ) and is therefore suppressed by a factor $1 / N$. Performing $(k-1)$ iterations only for the tree 
amplitudes, we conclude that

$$
e^{i H_{N} t / \hbar} A_{N}^{(p)} e^{-i H_{N} t / \hbar}=A_{t, N}^{(p)}+B_{t, N}^{(p), k}+\frac{1}{N} \sum_{n=1}^{k} Q_{t, N}^{(p), n}+R_{t, N}^{(p), k},
$$

where

$$
\begin{aligned}
B_{t, N}^{(p), k} & =\sum_{n=1}^{k-1} \int_{0}^{t} \ldots \int_{0}^{t_{n-1}} A_{N}^{(p+n)}\left(g_{t_{1}, \ldots, t_{n}}^{(n ; p)}\right) d t_{n} \ldots d t_{1}, \\
Q_{t, N}^{(p), n} & =\sum_{j>i=1}^{p+n-1} \int_{0}^{t} \ldots \int_{0}^{t_{n-1}} e^{i H_{N}^{0} t_{n} / \hbar} e^{-i H_{N} t_{n} / \hbar} H(n-1 ; p ; N) e^{i H_{N} t_{n} / \hbar} e^{-i H_{N}^{0} t_{n} / \hbar} d t_{n} \ldots d t_{1}, \\
R_{t, N}^{(p), k} & =\int_{0}^{t} \ldots \int_{0}^{t_{k-1}} e^{i H_{N}^{0} t_{n} / \hbar} e^{-i H_{N} t_{n} / \hbar} H(k, p ; N) e^{i H_{N} t_{n} / \hbar} e^{-i H_{N}^{0} t_{n} / \hbar} d t_{k} \ldots d t_{1},
\end{aligned}
$$

with

$$
H(s, p ; N):=\sum_{i<j}^{p+s-1} A_{N}^{(p+s-1)}\left(\left[w_{t_{s}}^{i j}, g_{t_{1}, \ldots, t_{s-1}}^{(s ; p)}\right]\right)
$$

Equation (2.17) is most easily verified as follows (think of $A_{N}^{(p+n-1)}\left(g_{t_{1}, \ldots, t_{n-1}}^{n-1, p}\right)$ as a $p+n-1$ particle operator replacing $A_{t, N}^{(p)}$ in $\left.(2.15)\right)$ :

$$
\begin{aligned}
& i \frac{\left[W_{t_{n}}, A_{N}^{(p+n-1)}\left(g_{t_{1}, \ldots, t_{n-1}}^{n-1, p}\right)\right]}{\hbar}=\frac{(N-(p+n-1))}{N} i \sum_{i=1}^{p+n-1} A_{N}^{(p+n)}\left(\left[w_{t_{n}}^{i p+n}, g_{t_{1}, \ldots, t_{n-1}}^{n-1, p}\right]\right)+ \\
& \frac{1}{N \hbar} \sum_{j>i=1}^{p+n-1} i A_{N}^{(p+n)}\left(\left[w_{t_{n}}^{i j}, g_{t_{1}, \ldots, t_{n-1}}^{n-1, p}\right]\right)=A_{N}^{(p+n)}\left(g_{t_{1}, \ldots, t_{n}}^{n, p}\right)+ \\
& \frac{1}{N \hbar} \sum_{j>i=1}^{p+n-1} i A_{N}^{(p+n)}\left(\left[w_{t_{n}}^{i j}, g_{t_{1}, \ldots, t_{n-1}}^{(n-1 ; p)}\right]\right)
\end{aligned}
$$

\subsection{Control of the expansion, small time, $\hbar$ fixed}

First, we prove a bound on the norm of $A_{N}^{(p+n)}\left(g_{t_{1}, \ldots, t_{n}}^{(n ; p)}\right)$

$$
\left\|A_{N}^{(p+n)}\left(g_{t_{1}, \ldots, t_{n}}^{(n ; p)}\right)\right\|_{\mathcal{H}^{(N)}} \leq \frac{2}{\hbar}(p+n-1)\|w\|_{\infty}\left\|A_{N}^{(p+n-1)}\left(g_{t_{1}, \ldots, t_{n-1}}^{(n-1 ; p)}\right)\right\|_{\mathcal{H}^{(N)}}
$$

This follows from the unitarity of the free time evolution and the boundedness of the interactions, $\left\|w^{i j}\right\|=\|w\|_{\infty}$. The bound (2.22) then yields recursively

$$
\begin{aligned}
\left\|A_{N}^{(p+n)}\left(g_{t_{1}, \ldots, t_{n}}^{(n ; p)}\right)\right\|_{\mathcal{H}^{(N)}} & \leq(p+n-1)(p+n-2) \cdots(p+1) p\left(\frac{2}{\hbar}\|w\|_{\infty}\right)^{n}\left\|A_{N}^{(p)}\right\|_{\mathcal{H}^{(N)}} \\
& \leq \frac{(p+n) !}{p !}\left(\frac{2}{\hbar}\|w\|_{\infty}\right)^{n}\left\|a^{(p)}\right\|_{\mathcal{H}^{(p)}},
\end{aligned}
$$

independently of all time indices. 
Considering the expansion (2.17), we have that

$$
\begin{aligned}
& \left\|\sum_{n=1}^{\infty} \int_{0}^{t} \cdots \int_{0}^{t_{n-1}} A_{N}^{(p+n)}\left(g_{t_{1}, \ldots, t_{n}}^{(n ; p)}\right) d t_{n} \cdots d t_{1}\right\|_{\mathcal{H}^{(N)}} \leq \\
& \sum_{n=1}^{\infty} \frac{t^{n}}{n !} \frac{(p+n) !}{p !}\left(\frac{2}{\hbar}\|w\|_{\infty} t\right)^{n}\left\|A_{N}^{(p)}\right\|_{\mathcal{H}^{(N)}} \leq 2^{p}\left\|a^{(p)}\right\|_{\mathcal{H}^{(p)}} \sum_{n=1}^{\infty}\left(\frac{4}{\hbar}\|w\|_{\infty} t\right)^{n}
\end{aligned}
$$

because $\frac{(p+n) !}{p ! n !} \leq 2^{n+p}$. The series on the R.S. of $(2.24)$ converges for $|t|<\left(\frac{4}{\hbar}\|w\|_{\infty}\right)^{-1}$. The third term in (2.17) is bounded similarly. Let

$$
A_{N, I}^{(p+n)}(i, j):=e^{i H_{N}^{0} t_{n} / \hbar} e^{-i H_{N} t_{n} / \hbar} A_{N}^{(p+n)}\left(\left[g_{t_{1}, \ldots, t_{n-1}}^{(n-1 ; p)}, w_{t_{n}}^{i j}\right]\right) e^{i H_{N}^{0} t_{n} / \hbar} e^{-i H_{N} t_{n} / \hbar}
$$

Then

$$
\begin{aligned}
& \frac{1}{N} \sum_{n=1}^{\infty}\left\|\sum_{i<j=1}^{p+n-1} \int_{0}^{t} \cdots \int_{0}^{t_{n-1}} A_{N, I}^{(p+n)}(i, j) d t_{n} \cdots d t_{1}\right\|_{\mathcal{H}^{(N)}} \leq \\
& \frac{1}{N} \sum_{n=1}^{\infty} \frac{(p+n-1)^{2}}{2} \frac{2}{\hbar}\|w\| \int_{0}^{t} \cdots \int_{0}^{t_{n-1}}\left\|A_{N}^{(p+n-1)}\left(g_{t_{1}, \ldots, t_{n-1}}^{(n-1 ; p)}\right)\right\|_{\mathcal{H}^{(N)}} d t_{n} \ldots d t_{1} \leq \\
& \leq \frac{1}{N} \sum_{n=1}^{\infty}(p+n-1)^{2}(p+n-2) \cdots p\left(\frac{2}{\hbar}\|w\|_{\infty}\right)^{n-1} \frac{2}{\hbar}\|w\|_{\infty}\left\|A^{(p)}\right\|_{\mathcal{H}^{(N)}} \frac{|t|^{n}}{n !} \leq \\
& \leq \frac{\left\|a^{(p)}\right\|_{\mathcal{H}^{(p)}}}{N} \sum_{n=1}^{\infty}\left(\frac{(p+n) !}{n ! p !}\left(\frac{2}{\hbar}\|w\|_{\infty} t\right)^{n} \leq \frac{\left\|a^{(p)}\right\|_{\mathcal{H}^{(p)}}}{N} 2^{p} \sum_{n=1}^{\infty}\left(\frac{4}{\hbar}\|w\|_{\infty}|t|\right)^{n}\right.
\end{aligned}
$$

Therefore

$$
\left\|\frac{1}{N} \sum_{n=1}^{k} Q_{t, N}^{(p), n}\right\|_{\mathcal{H}^{(N)}} \leq \frac{\left\|a^{(p)}\right\|_{\mathcal{H}^{(p)}}}{N} 2^{p} \sum_{n=1}^{\infty}\left(\frac{4}{\hbar}\|w\|_{\infty} \mid t !\right)^{n} .
$$

The remainder term in (2.17) clearly vanishes, as $k \rightarrow \infty$. To summarize, we have proven the following result.

Proposition 2.2 . Let $|t|<\left(\frac{4}{\hbar}\|w\|_{\infty}\right)^{-1}$. Then

$$
e^{i H_{N} t / \hbar} A_{N}^{(p)} e^{-i H_{N} t / \hbar}=A_{t}^{(p)}+\sum_{n=1}^{\infty} \int_{0}^{t} \ldots \int_{0}^{t_{n-1}} A_{N}^{(p+n)}\left(g_{t_{1}, \ldots, t_{n}}^{(n ; p)}\right) d t_{n} \ldots d t_{1}+O(1 / N)
$$

\subsection{Convergence for all times, $\hbar$ fixed}

We assume that the statement of Theorem 1.1 holds up to some time $T$ independent of $p$, i.e.,

$$
\lim _{N \rightarrow \infty}\left\langle\Psi_{N, 0}, e^{i H_{N} T / \hbar} A_{N}^{(p)} e^{-i H_{N} T / \hbar} \Psi_{N, 0}\right\rangle_{\mathcal{H}^{(N)}}=a^{(p)}\left(\psi_{p, T}\right)
$$


Let us proceed one step further in time, with $t<\left(4\|w\|_{\infty} / \hbar\right)^{-1}$. On account of $(2.17)$, we have that

$$
\begin{aligned}
& e^{i H_{N}(T+t) / \hbar} A_{N}^{(p)} e^{-i H_{N}(T+t) / \hbar}= \\
& e^{i H_{N} T / \hbar} e^{-i H_{N} t / \hbar} A_{N}^{(p)} e^{-i H_{N} t / \hbar} e^{-i H_{N} T / \hbar}=e^{i H_{N} T / \hbar} A_{N}^{(p)}(t) e^{-i H_{N} T / \hbar} \\
& +\sum_{n=1}^{N} \int_{0}^{t} \ldots \int_{0}^{t_{n-1}} e^{i H_{N} T / \hbar} A_{N}^{(p+n)}\left(g_{t_{1}, \ldots, t_{n}}^{(n ; p)}\right) e^{-i H_{N} T / \hbar} d t_{n} \ldots d t_{1}+O(1 / N)
\end{aligned}
$$

This expansion is norm convergent, by $(2.17)$ and the unitarity of $e^{i H_{N} T / \hbar}$. Taking expectation values in $\Psi_{N, 0}$ we get, as above,

$$
\begin{aligned}
& \left\langle\Psi_{N, 0}, e^{i H_{N}(T+t) / \hbar} A_{N}^{(p)} e^{-i H_{N}(T+t) / \hbar} \Psi_{N, 0}\right\rangle_{\mathcal{H}^{(N)}}= \\
& =\left\langle\Psi_{N, 0}, e^{i H_{N} T / \hbar} A_{t}^{(p)} e^{-i H_{N} T / \hbar} \Psi_{N, 0}\right\rangle_{\mathcal{H}^{(N)}}+ \\
& \sum_{n=1}^{N} \int_{0}^{t} \ldots \int_{0}^{t_{n-1}}\left\langle\Psi_{N, 0}, e^{i H_{N} T / \hbar} A_{N}^{(p+n)}\left(g_{t_{1}, \ldots, t_{n}}^{(n ; p)}\right) e^{-i H_{N} T / \hbar} \Psi_{N, 0}\right\rangle_{\mathcal{H}^{(N)}} d t_{n} \ldots d t_{1} \\
& +O(1 / N)
\end{aligned}
$$

Hence, by the inductive assumption and the norm convergence of the series

$$
\begin{aligned}
& \lim _{N \rightarrow \infty}\left\langle\Psi_{N}, e^{i H_{N}(T+t) / \hbar} A_{N}^{(p)} e^{-i H_{N}(T+t) / \hbar} \Psi_{N}\right\rangle_{\mathcal{H}^{(N)}}=a^{(p)}\left(e^{i t \Delta / \hbar} \psi_{T}\right)+ \\
& \sum_{n=1}^{\infty} \lim _{N \rightarrow \infty} \int_{0}^{t} \ldots \int_{0}^{t_{n-1}}\left\langle\Psi_{N, t}, e^{i H_{N} T / \hbar} A_{N}^{(p+n)}\left(g_{t_{1}, \ldots, t_{n}}^{(n ; p)}\right) e^{-i H_{N} T / \hbar} \Psi_{N, t}\right\rangle_{\mathcal{H}^{(N)}} d t_{n} \ldots d t_{1}
\end{aligned}
$$

We postpone to Section 3, below, the proof that actually

$$
\begin{aligned}
& a^{(p)}\left(e^{i t \Delta / \hbar} \psi_{T}\right)+ \\
& \lim _{N \rightarrow \infty} \sum_{n=1}^{\infty} \int_{0}^{t} \ldots \int_{0}^{t_{n-1}}\left\langle\Psi_{N, t}, A_{N}^{(p+n)}\left(g_{t_{1}, \ldots, t_{n}}^{(n ; p)}\right) \Psi_{N, t}\right\rangle_{\mathcal{H}^{(N)}} d t_{n} \ldots d t_{1} \\
& =a^{(p)}\left(\psi_{t+T}\right),
\end{aligned}
$$

and this ensures that the convergence is global in time.

\subsection{Control of the expansion, uniformity with respect to $\hbar$}

Given a symbol $\tau(x, \xi) \in \mathcal{A}_{\sigma, p}$, we denote by $T$ the corresponding Weyl operator. Its action on vectors $\psi \in \mathcal{S}\left(\mathbb{R}^{3 p}\right)$ is given by

$$
(T \psi)(x)=\frac{1}{\hbar^{3 p}} \int_{\mathbb{R}^{3 p}} \int_{\mathbb{R}^{3 p}} \tau[(x+y) / 2, \xi] e^{i\langle(x-y), \xi\rangle / \hbar} \psi(y) d y d \xi
$$

In general, $T$ is a semiclassical pseudodifferential operator. Let us recall some relevant results (see e.g. [Ro]). 
1. If $\widehat{\tau} \in L^{1}\left(\mathbb{R}^{3 p} \times \mathbb{R}^{3 p}\right)$ then $T$ extends to a continuous operator on $L^{2}\left(\mathbb{R}^{3 p}\right)$ with $\|T\| \leq\|\widehat{\tau}\|_{L^{1}}$; hence $\|T\|_{L^{2} \rightarrow L^{2}} \leq\|T\|_{\sigma}:=\|\tau\|_{\sigma}$, where $\widehat{\tau}$ is the Fourier transform of $\tau$, and

$$
\|\tau\|_{\sigma}:=\int_{\mathbb{R}^{6 p}}|\hat{\tau}(s)| e^{\sigma|s|} d s
$$

Obviously, $\|\widehat{\tau}\|_{L^{1}} \leq\|\tau\|_{\sigma}$.

2. If $w \in \mathcal{A}_{\sigma, p}, g \in \mathcal{A}_{\sigma, p}$, then the symbol of the commutator $[W, G] / i \hbar$ is the Moyal bracket $\{w, g\}_{M}$. Hence the multiple commutator $\left[W,[W, \ldots[W, G] \ldots] /(i \hbar)^{n}\right.$ has as its symbol the multiple Moyal bracket $\left\{w,\left\{w, \ldots,\{w, g\}_{M} \ldots\right\}_{M}\right.$.

We recall that, given $g, g^{\prime} \in \mathcal{A}_{\sigma, p}$, their Moyal bracket $\left\{g, g^{\prime}\right\}_{M}$ is defined as

$$
\left\{g, g^{\prime}\right\}_{M}=g \# g^{\prime}-g^{\prime} \# g,
$$

where \# is the composition of Weyl symbols. In the Fourier transform representation the explicit expression of the Moyal bracket is (see e.g. [Fo],§3.4):

$$
\left(\left\{g, g^{\prime}\right\}_{M}\right)^{\wedge}(s)=\frac{2}{\hbar} \int_{\mathbb{R}^{2 n}} \widehat{g}\left(s^{1}\right) \widehat{g^{\prime}}\left(s-s^{1}\right) \sin \left[\hbar\left(s-s^{1}\right) \wedge s^{1} / 2\right] d s^{1},
$$

where, given two vectors $s=(v, w)$ and $s^{1}=\left(v^{1}, w^{1}\right), s \wedge s^{1}:=\left\langle w, v_{1}\right\rangle-\left\langle v, w_{1}\right\rangle$.

3. If the observable $T$ has symbol $\tau(x, \xi)$, then the Heisenberg observable $T_{t}$ has symbol $\left(\tau \circ \Phi_{t}^{0}\right)(x, \xi)$. Here $\Phi_{t}^{0}(x, \xi)=(x+\xi t, \xi)$ is the free flow with initial conditions $(x, \xi)$. In particular, $\left(\tau \circ \Phi_{t}^{0}\right)(x, \xi) \in \mathcal{A}_{\sigma, p}$ whenever $\tau \in \mathcal{A}_{\sigma, p}$.

Under the present assumptions, it can be proven, starting from the expression (2.31) (see [BGP], Lemma 3.2 ), that the following estimate on the Moyal bracket holds:

$$
\left\|\{w, g\}_{M}\right\|_{\sigma-\delta} \leq \frac{1}{e^{2} \delta^{2}}\|w\|_{\sigma}\|g\|_{\sigma}, \quad 0<\delta<\sigma
$$

For the convenience of the reader we reproduce here the proof of (2.32). Since $\left(s-s^{1}\right) \wedge s^{1}=$

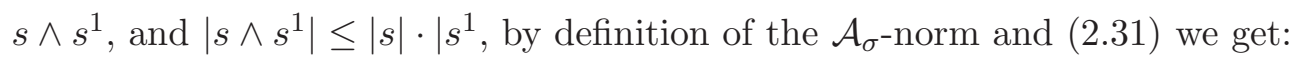

$$
\begin{aligned}
& \left\|\left(\left\{g, g^{\prime}\right\}_{M}\right)\right\|_{\sigma-\delta}= \\
& \frac{2}{\hbar} \int_{\mathbb{R}^{6 p}} e^{(\sigma-\delta)|s|} d s \int_{\mathbb{R}^{6 p}}\left|\hat{g}\left(s^{1}\right) \hat{g}^{\prime}\left(s-s^{1}\right) \sin \left(\hbar\left(s-s^{1}\right) \wedge s^{1}\right) / 2\right| d s^{1} \\
& \leq \frac{2}{\hbar} \int_{\mathbb{R}^{6 p}} d s \int_{\mathbb{R}^{6 p}} e^{(\sigma-\delta)\left(|s|+\left|s^{1}\right|\right)} \mid \hat{g}(s) \hat{g}^{\prime}\left(s^{1}\right) \sin \left(\hbar\left(s \wedge s^{1}\right) / 2 \mid d s^{1}\right. \\
& \leq \int_{\mathbb{R}^{6 p}} e^{(\sigma-\delta)|s|}|\hat{g}(s)| d s \int_{\mathbb{R}^{6 p}} e^{(\sigma-\delta)\left|s^{1}\right|}\left|\hat{g}^{\prime}\left(s^{1}\right) s \wedge s^{1}\right| d s^{1} \\
& \leq \int_{\mathbb{R}^{6 p}} e^{(\sigma-\delta)|s|}|\hat{g}(s)||s| d s \int_{\mathbb{R}^{6 p}} e^{(\sigma-\delta)\left|s^{1}\right|}\left|\hat{g}^{\prime}\left(s^{1}\right) s \| s^{1}\right| \mid d s^{1}
\end{aligned}
$$


whence the assertion because $x e^{-\delta x} \leq \frac{1}{e \delta}, \forall x>0, \forall \delta>0$.

Let now $g_{r}:=\left\{g_{r-1}, w\right\}_{M}, r>1 ; g_{1}=\{g, w\}_{M}$. Then, applying (2.32) $r$ times, we can write:

$$
\left\|g_{r}\right\|_{\sigma-r \delta} \leq\left(\frac{1}{e^{2} \delta^{2}}\right)^{r}\|w\|_{\sigma}^{r}\|g\|_{\sigma}
$$

These results immediately yield the following bound.

Lemma 2.1 . Let the operator $a^{(p)}$ be the Weyl quantization of a symbol $\tau_{a}(x, \xi) \in \mathcal{A}_{\sigma, p}$ for some $\sigma>0$. Then there is $L(p)>0$ independent of $\hbar$ such that

$$
\left\|A_{N}^{(p+n)}\left(g_{t_{1}, \ldots, t_{n}}^{(n ; p)}\right)\right\|_{\mathcal{H}^{(N)}} \leq L^{n} n !^{3}\left(2\|w\|_{\sigma}\right)^{n}\left\|a^{(p)}\right\|_{\sigma}
$$

\section{Proof}

Denote by $\mathcal{G}_{t, t_{1}, \ldots, t_{n}}^{(n, p)}$ the symbol of $g_{t_{1}, \ldots, t_{n}}^{(n ; p)}$. Using definition (2.16) and the estimate $(2.32)$ we get the uniform estimate corresponding to (2.23):

$$
\left\|\mathcal{G}_{t, t_{1}, \ldots, t_{n} ; N}^{(n, p)}\right\|_{\sigma-n \delta_{n}} \leq \frac{2(p+n-1)}{e^{2} \delta_{n}^{2}}\|w\|_{\sigma}\left\|\mathcal{G}_{t, t_{1}, \ldots, t_{n-1}}^{(n-1, p)}\right\|_{\sigma}, \quad 0<\delta_{n}<\sigma
$$

The recursive definition (2.16) allows us to use the recursive estimate (2.33). We get

$$
\left\|\mathcal{G}_{t, t_{1}, \ldots, t_{n}}^{(n, p)}\right\|_{\sigma-n \delta_{n}} \leq 2^{n}\left(e^{2} \delta_{n}^{2}\right)^{-n} \frac{(p+n) !}{p !}\|w\|_{\sigma}^{n}\left\|a^{(p)}\right\|_{\sigma}
$$

Setting $\delta_{n}:=\frac{1}{2 n}$ we get the bound (2.34) on account of the majorizations

$$
\left\|A_{N}^{(p+n)}\left(g_{t_{1}, \ldots, t_{n}}^{(n ; p)}\right)\right\|_{\mathcal{H}^{(N)}} \leq\left\|\mathcal{G}_{t, t_{1}, \ldots, t_{n}}^{(n, p)}\right\|_{\sigma / 2}, \quad\|w\|_{L^{2} \rightarrow L^{2}}=\|w\|_{\infty} \leq\|w\|_{\sigma}
$$

This proves the Lemma.

\section{Remark}

The uniform control in $\hbar$ introduces an extra $n !^{2}$ divergence with respect to the fixed- $\hbar$ estimate (2.23).

We now obtain uniform estimates of the three terms in expansion (2.17).

Lemma 2.2. There exist constants $M_{1}>0, M_{2}>0, M_{3}>0, L_{1}>0, L_{2}>0, L_{3}>0$, independent of $(\hbar, t)$ and $N$, such that

$$
\begin{aligned}
\left\|B_{t, N}^{(p), k}\right\|_{\mathcal{H}^{(N)}} & \leq M_{1}\left\|a^{(p)}\right\|_{\sigma} \sum_{n=1}^{k} L_{1}^{n} n !^{2}\left(\|w\|_{\sigma} t\right)^{n} \\
\left\|Q_{t, N}^{(p), n}\right\|_{\mathcal{H}^{(N)}} & \leq M_{3}\left\|a^{(p)}\right\|_{\sigma} L_{2}^{n} n !^{2}\left(\|w\|_{\sigma} t\right)^{n} \\
\left\|R_{t, N}^{(p), k}\right\|_{\mathcal{H}^{(N)}} & \leq M_{3}\left\|a^{(p)}\right\|_{\sigma} L_{3}^{k} k !^{2}\left(\|w\|_{\sigma} t\right)^{k}
\end{aligned}
$$




\section{Proof}

Inserting the estimate $(2.34)$ in the expressions $(2.18,2.19,2.20)$ we get, on account of unitarity of $U_{0}(t)$ :

$$
\begin{aligned}
\left\|B_{t, N}^{(p), k}\right\|_{\mathcal{H}^{(N)}} & \leq\left\|a^{(p)}\right\|_{\sigma} \sum_{n=1}^{k}\left(2 L\|w\|_{\sigma}\right)^{n} n !^{3} \int_{0}^{t} \ldots \int_{0}^{t_{n-1}} d t_{n} \ldots d t_{1} \\
& \leq\left\|a^{(p)}\right\|_{\sigma} \sum_{n=1}^{k}\left(2 L\|w\|_{\sigma}|t|\right)^{n} n !^{2} .
\end{aligned}
$$

The last inequality comes from performing the time integrations, which are majorized by a factor $|t|^{n} / n$ ! in $(2.37,2.38)$ and by a factor $|t|^{k} / k$ ! in $(2.39)$ (proven with the help of the same argument). This proves the lemma.

Using this result, we can easily prove the uniform version of the expansion (2.17).

Proposition 2.3 . Let $\epsilon:=\|w\|_{\infty} t$. Then, in the same assumption of Lemma 2.1 on the operator $a^{(p)}$, there exists $k=k(\epsilon), \Lambda=\Lambda(\epsilon)$ such that

$$
e^{i H_{N} t / \hbar} A_{N}^{(p)} e^{-i H_{N} t \hbar}=A_{t, N}^{(p)}+B_{t, N}^{(p), k}+R_{t, N}^{(p), k}+\frac{\Lambda}{N},
$$

where

$$
B_{t, N}^{(p), k}=\sum_{n=1}^{k} \int_{0}^{t} \ldots \int_{0}^{t_{n-1}} A_{N}^{(p+n)}\left(g_{t_{1}, \ldots, t_{n}}^{(n ; p)}\right) d t_{n} \ldots d t_{1} .
$$

Here $B_{t, N}^{(p), k}$ fulfills the majorization (2.37), and

$$
\left\|R_{t, N}^{(p), k}\right\|_{\mathcal{H}^{(N)}} \leq M_{3} e^{-L_{3} / \sqrt{\epsilon}}
$$

\section{Proof}

The estimate (2.38) and a standard Nekhoroshev-type argument show that the choice

$$
k(\epsilon):=\frac{1}{\sqrt{\epsilon}}=\frac{1}{\|w\|_{\infty} t}
$$

minimizes the divergence of $R_{t, N}^{(p), k}$. A straightforward computation then yields (2.42). By definition of $Q_{t, N}^{(p), N}$ we get the uniform version of the estimate (2.21), whence

$$
\Lambda(\epsilon):=p 2^{p}\left\|a^{(p)}\right\|_{\sigma} \sum_{n=1}^{k(\epsilon)} n !^{2}(2 \epsilon)^{n} \leq p 2^{p} \epsilon^{-1 / 2}(e / 2)^{-1 / \sqrt{\epsilon}}
$$




\section{Connection with the Hartree equation and proof of the theorems}

We wish to prove that the representation of the evolution obtained in Proposition 2.2 coincides with the evolution generated by the Hartree equation in the limit $N \rightarrow \infty$.

For this purpose, we recall that the Hartree equation is Hamiltonian. We define the functional

$$
\mathcal{H}(\psi, \bar{\psi})=-\frac{\hbar^{2}}{2} \int_{\mathbb{R}^{3}}|\nabla \psi(x)|^{2} d x+\mathcal{W}(\psi, \bar{\psi}),
$$

for $\psi \in H^{1}\left(\mathbb{R}^{3}\right)$, where

$$
\mathcal{W}(\psi, \bar{\psi})=\frac{1}{2} \int_{\mathbb{R}^{3} \times \mathbb{R}^{3}} \overline{\psi(x) \psi(y)} w(x-y) \psi(x) \psi(y) d x d y
$$

If $\psi(x), \overline{\psi(y)}$ are considered as canonical variables with Poisson brackets

$$
\{\psi(x), \overline{\psi(y)})\}=i \hbar \delta(x-y), \quad\{\psi(x), \psi(y)\}=\{\overline{\psi(x)}, \overline{\psi(y)}\}=0
$$

then (3.1) is the Hamiltonian functional generating a time evolution of functionals on phase space equivalent to the Hartree equation. Namely, if $\mathcal{A}(\psi)$ is a functional and $\mathcal{A}_{t}$ denotes its time evolution, one has that

$$
\partial_{t} \mathcal{A}_{t}(\psi)=\frac{1}{\hbar}\left\{\mathcal{H}, \mathcal{A}_{t}\right\}(\psi)
$$

Choosing $\mathcal{A}=\langle\phi, \psi\rangle, \phi \in C_{0}^{\infty}\left(\mathbb{R}^{3}\right)$, then $\mathcal{A}_{t}(\psi)=\mathcal{A}\left(\psi_{t}\right)$, where $\psi_{t}$ is a solution of the Hartree equation

$$
i \hbar \partial_{t} \psi_{t}=-\frac{\hbar^{2}}{2} \Delta \psi_{t}+\left(w *\left|\psi_{t}\right|^{2}\right) \psi_{t}
$$

Define the free flow $\Phi_{t}^{0}(\mathcal{A}):=\mathcal{A}_{t}$ of $\mathcal{A}$ by

$$
\mathcal{A}_{t}=\mathcal{A}\left(e^{i \Delta t / \hbar} \psi\right)
$$

and denote by $\Phi_{t}(\mathcal{A})$ the interacting flow. Formally, the interacting flow is given by the Lie expansion in the interaction representation (analogous to the Schwinger-Dyson expansion of Section 2.2). Indeed we have the following result:

Lemma 3.3. $\Phi_{t}(\mathcal{A})$ admits the formal expansion

$$
\Phi_{t}(\mathcal{A})=\mathcal{A}_{t}+\Phi_{t}^{0}\left(\sum_{n=1}^{\infty}\left(\frac{1}{\hbar}\right)^{n} \int_{0}^{t} \ldots \int_{0}^{t_{n}}\left\{\mathcal{W}_{t_{n}} \ldots\left\{\mathcal{W}_{t_{1}}, \mathcal{A}\right\} \ldots\right\} d t_{n} \ldots d t_{1}\right)
$$


Proof. To see this, we consider the dynamics in the interaction picture. We set

$$
\tilde{\mathcal{A}}_{t}:=\Phi_{t} \circ \Phi_{-t}^{0}(\mathcal{A})
$$

Then

$$
\partial_{t} \tilde{\mathcal{A}}_{t}=\frac{\left\{\tilde{\mathcal{A}}_{t}, \mathcal{W}_{t}\right\}}{\hbar}
$$

where $\mathcal{W}_{t}:=\Phi_{t}^{0}(\mathcal{W})$ is the free evolution of $\mathcal{W}$. After integrating in time we get

$$
\tilde{\mathcal{A}}_{t}=\mathcal{A}+\int_{0}^{t} \frac{\left\{\mathcal{W}_{s}, \mathcal{A}\right\}}{\hbar} d s
$$

whence

$$
\Phi_{t}(\mathcal{A})=\mathcal{A}_{t}+\Phi_{t}^{0}\left(\int_{0}^{t} \frac{\left\{\mathcal{W}_{s}, \mathcal{A}\right\}}{\hbar} d s\right)
$$

Iterating this identity, we obtain the series (3.4), and this concludes the proof of the Lemma. The desired identification is based on the following proposition

Proposition 3.4 . Let $\psi \in H^{1}\left(\mathbb{R}^{3}\right)$, and let $\Psi$ be a product state, i.e.

$$
\Psi\left(x_{1}, \ldots, x_{l}\right)=\prod_{s=1}^{l} \psi\left(x_{s}\right)
$$

Then, for all $N \geq p$,

$$
\begin{gathered}
g_{t_{1}, \ldots, t_{n}}^{(n ; p)}(\psi):=\frac{(p+n)^{p+n}}{(p+n) !}\left\langle\Psi_{n+p}, A_{p+n}^{(p+n)}\left(g_{t_{1}, \ldots, t_{n}}^{(n ; p)}\right) \Psi_{n+p}\right\rangle_{\mathcal{H}^{(n+p)}}= \\
\left(\frac{1}{\hbar}\right)^{n}\left\{\mathcal{W}_{t_{n}} \ldots\left\{\mathcal{W}_{t_{1}}, \mathcal{A}\right\} \ldots\right\}(\psi),
\end{gathered}
$$

where

$$
\mathcal{A}=a^{(p)}(\psi):=\int \overline{\psi\left(x_{1}\right)} \cdots \overline{\psi\left(x_{p}\right)} \alpha^{(p)}\left(x_{1}, \ldots, x_{p} ; y_{1}, \ldots, y_{p}\right) \overline{\psi\left(y_{1}\right)} \cdots \overline{\psi\left(y_{p}\right)} \prod_{k=1}^{p} d x_{k} d y_{k}
$$

Proof. We have that

$$
\left.\Phi_{t} \circ \Phi_{-t}^{0}\left(\left\{\mathcal{W}_{t}, \mathcal{A}\right\} / \hbar\right)\right|_{t=0}=\{\mathcal{W}, \mathcal{A}\} / \hbar=\left.\partial_{t} \tilde{\mathcal{A}}\right|_{t=0}
$$

Define the projection $\rho:=|\psi\rangle\langle\psi|$. Then, denoting $\tilde{\rho}_{t}:=\Phi_{t} \circ \Phi_{-t}^{0}(\rho)$, we have that, for all $n \geq 1$,

$$
\tilde{\mathcal{A}}_{t}=\operatorname{Tr}\left(\mathcal{A} \tilde{\rho}_{t}^{\otimes n}\right) ;\left.\quad \partial_{t} \tilde{\mathcal{A}}\right|_{t=0}=\left.\partial_{t} \operatorname{Tr}\left(\mathcal{A} \tilde{\rho}_{t}^{\otimes n}\right)\right|_{t=0}
$$

Therefore, in the interaction picture

$$
\partial_{t} \tilde{\rho}_{t}=\operatorname{Tr}\left(\tilde{\rho}_{t}^{\otimes 2} \mathcal{W}_{t}^{12}-\mathcal{W}_{t}^{12} \tilde{\rho}_{t}^{\otimes 2}\right) / \hbar
$$


and in the same way we get

$$
\frac{1}{\hbar}\left\{\mathcal{W}_{t}, \mathcal{A}\right\}=\sum_{i=1}^{n} \operatorname{Tr}\left(\left(\mathcal{W}_{t}^{i n+1} \mathcal{A}-\mathcal{A} \mathcal{W}_{t}^{i n+1}\right) \rho^{\otimes n+1}\right)
$$

It is then easy to check that

$$
\begin{aligned}
\frac{1}{\hbar^{n}}\left\{\mathcal{W}_{t_{n}} \ldots\left\{\mathcal{W}_{t_{1}}, \mathcal{A}\right\} \ldots\right\}(\psi) & =\operatorname{Tr}\left(g_{t_{1}, \ldots, t_{n}}^{(n ; p)} \cdot \rho^{\otimes n+p}\right) \\
& =\frac{(p+n)^{p+n}}{(p+n) !}\left\langle\Psi_{n+p}, A_{p+n}^{(p+n)}\left(g_{t_{1}, \ldots, t_{n}}^{(n ; p)}\right) \Psi_{n+p}\right\rangle_{\mathcal{H}^{(n+p)}} \\
& =g_{t_{1}, \ldots, t_{n}}^{(n ; p)}(\psi),
\end{aligned}
$$

and this concludes the proof of the Proposition.

We are now in a position to prove our main results.

\section{Proof of Theorem 1.1}

Consider the expectation value of the expansion (2.26) in a coherent (i.e., product) state:

$$
\begin{aligned}
& \left\langle\Psi_{N}, e^{i H_{N} t / \hbar} A_{N}^{(p)} e^{-i H_{N} t / \hbar} \Psi_{N}\right\rangle_{\mathcal{H}^{(N)}}=\left\langle\Psi_{N}, A_{t, N}^{(p)} \Psi_{N}\right\rangle_{\mathcal{H}^{(N)}}+ \\
& \sum_{n=1}^{N} \int_{0}^{t} \ldots \int_{0}^{t_{n-1}}\left\langle\Psi_{N}, A_{N}^{(p+n)}\left(g_{t_{1}, \ldots, t_{n}}^{(n ; p)}\right) \Psi_{N}\right\rangle_{\mathcal{H}^{(N)}} d t_{n} \cdots d t_{1}+O(1 / N) .
\end{aligned}
$$

By definition of $\Psi_{N}$,

$$
\begin{aligned}
& \frac{N^{p}}{N(N-1) \ldots(N-p+1)}\left\langle\Psi_{N}, e^{i H_{N} t / \hbar} A_{N} e^{-i H_{N} t / \hbar} \Psi_{N}\right\rangle_{\mathcal{H}^{(N)}}=\left\langle\Psi_{p}, a_{t}^{(p)} \Psi_{p}\right\rangle_{\mathcal{H}^{(p)}}+ \\
& +\sum_{n=1}^{\infty} \int_{0}^{t} \ldots \int_{0}^{t_{n-1}}\left\langle\Psi_{n+p}, g_{t_{1}, \ldots, t_{n}}^{(n ; p)} \Psi_{n+p}\right\rangle_{\mathcal{H}^{(n+p)}} d t_{n} \cdots d t_{1}+O(1 / N) .
\end{aligned}
$$

Since the series is norm- convergent, the limits $N \rightarrow \infty$ and $n \rightarrow \infty$ can be interchanged.

Then

$$
\begin{aligned}
\lim _{N \rightarrow \infty} & \left\langle\Psi_{N}, e^{i H_{N} t / \hbar} A_{N} e^{-i H_{N} t / \hbar} \Psi_{N}\right\rangle_{\mathcal{H}^{(N)}}=\left\langle\Psi_{p}, a_{t}^{(p)} \Psi_{p}\right\rangle_{\mathcal{H}^{(p)}}+ \\
& \sum_{n=1}^{\infty} \int_{0}^{t} \ldots \int_{0}^{t_{n-1}}\left\langle\Psi_{n+p}, g_{t_{1}, \ldots, t_{n}}^{(n ; p)} \Psi_{n+p}\right\rangle_{\mathcal{H}^{(n+p)}} d t_{n} \cdots d t_{1}=a^{(p)}\left(\psi_{t}\right),
\end{aligned}
$$

where the last equality follows from formula (3.5).

\section{Proof of Theorem 1.2}

If, instead of (2.26), the representation (2.40) is considered, the above argument yields

$$
\begin{aligned}
& \frac{N^{p}}{N(N-1) \ldots(N-p+1)}\left\langle\Psi_{N}, e^{i H_{N} t / \hbar} A_{N}^{(p)} e^{-i H_{N} t / \hbar} \Psi_{N}\right\rangle=\left\langle\Psi_{p}, a_{t}^{(p)} \Psi_{p}\right\rangle+\left\langle\Psi_{N}, R_{t, N}^{k, p} \psi_{N}\right\rangle \\
& +\sum_{n=1}^{k(\epsilon)} \int_{0}^{t} \ldots \int_{0}^{t_{n-1}}\left\langle\Psi_{n+p}, g_{t_{1}, \ldots, t_{n}}^{(n ; p)} \Psi_{n+p}\right\rangle d t_{n} \cdots d t_{1}=a^{(p)}\left(\psi_{t}\right)+O\left(e^{-1 / \sqrt{\epsilon}}\right) .
\end{aligned}
$$


Given any bounded operator $A$ on $L^{2}\left(\mathbb{R}^{3 l}\right)$ with (Weyl) symbol $\sigma_{A}(x, \xi): \mathcal{S}\left(\mathbb{R}^{6 l}\right) \rightarrow \mathbb{R}$, where $\mathcal{S}$ is the Schwartz space of rapidly decreasing functions, its matrix elements can be expressed in terms of the symbol and of the Wigner function by the following well known formula (see e.g.[Fo]):

$$
\langle\Psi, A \Psi\rangle=\int_{\mathbb{R}^{3 l} \times \mathbb{R}^{3 l}} \sigma_{A}(x, \xi) W_{\Psi}(x, \xi) d x d \xi
$$

where $W_{\Psi}(x, \xi)$ is the Wigner function of the state $\Psi$. Therefore, in our case

$$
\frac{N^{p}}{N(N-1) \ldots(N-p+1)}\left\langle\Psi_{N}, A_{N}(t) \Psi_{N}\right\rangle=\int_{\mathbb{R}^{3 p} \times \mathbb{R}^{3 p}} \sigma_{A}\left(X_{p}, \Xi_{p}\right) W_{N}^{\Psi_{N}}\left(X_{p}, \Xi_{p}, t\right) d X_{p} d \Xi_{p},
$$

where $W_{N}^{\Psi_{N}}\left(X_{p}, \Xi_{p}, t\right)$ is the Wigner function corresponding to the time evolution, $e^{i H_{N} t / \hbar} \Psi_{N}$, of the product state $\Psi_{N, 0}=\psi\left(x_{1}\right) \ldots \psi\left(x_{N}\right)$. The $N-p$ variables $\left(X_{N-p}, \Xi_{N-p}\right)$ are integrated out. By (3.7) and (1.13), we can take the $N \rightarrow \infty$ limit and write

$$
\begin{array}{r}
\int_{\mathbb{R}^{3 p} \times \mathbb{R}^{3 p}} \sigma_{A}\left(X_{p}, \Xi_{p}\right) W_{N}^{\Psi_{N}}\left(X_{p}, \Xi_{p}, t\right) d X_{p} d \Xi_{p}= \\
\int_{\mathbb{R}^{3 p} \times \mathbb{R}^{3 p}} \sigma_{A}\left(X_{p}, \Xi_{p}\right) \prod_{l=1}^{p} W_{\psi}\left(x_{l}, \xi_{l} ; t\right) d X_{p} d \Xi_{p}+O\left(e^{-1 / \sqrt{\epsilon}}\right) .
\end{array}
$$

Since this formula holds for any $\sigma_{A}\left(X_{p}, \Xi_{p}\right) \in \mathcal{S}\left(\mathbb{R}^{3 p} \times \mathbb{R}^{3 p}\right) \cap \mathcal{A}_{\sigma, p}$, the assertion is proved.

\section{Proof of formula (2.29).}

By (3.5), we have that

$$
\begin{aligned}
& \left\langle\Psi_{N}, e^{i H_{N} T / \hbar} A_{N}^{(p+n)}\left(g_{t_{1}, \ldots, t_{n}}^{(n ; p)}\right) e^{-i H_{N} T / \hbar} \Psi_{N}\right\rangle_{\mathcal{H}^{(N)}}= \\
& \left(\frac{1}{\hbar}\right)^{n}\left\{\mathcal{W}_{t_{n}} \ldots\left\{\mathcal{W}_{t_{1}}, \mathcal{A}\right\} \ldots\right\}\left(\psi_{T}\right)
\end{aligned}
$$

which yields formula (2.29), by Lemma 3.3, on account of the uniform convergence of the series. 


\section{References}

[BGM] C. Bardos, F. Golse, N. Mauser Weak coupling limit of the N-particle Schrödinger equation, Methods Appl.Anal. 2, 275-293, 2000

[BEGMY] C. Bardos, L. Erdös, F. Golse, N. Mauser and H-T Yau Derivation of the SchrödingerPoisson equation from the quantum N-body problem C.R. Acad. Sci. Paris 334 no. 6, $515-520,2002$

[BGP] D. Bambusi, S. Graffi, T. Paul, Normal Forms Estimates and Quantization Formulae Comm. Math. Phys. 207, 173-195, 1999

[BH] W. Braun, K. Hepp, The Vlasov Dynamics and Its Fluctuations in the 1/N Limit of Interacting Classical Particles, Comm. Math. Phys. 56, 101-113, 1977

[Do] R. L. Dobrushin, Vlasov equations, Sov.J.Funct.An. 13, 115-119, 1979

[EY] L. Erdos and H.-T. Yau, Derivation of the nonlinear Schrödinger equation from a many body Coulomb system, http://www.citebase.org/cgibin/citations?id=oai:arXiv.org:math-ph/0111042, 2001

[Fo] G.Folland Harmonic Analysis in Phase Space, Princeton University Press 1989

[GMP] S.Graffi, A.Martinez, M.Pulvirenti, Mean field approximation of quantum systems and classical limit, Mathematical Methods and Models in Applied Sciences 13, 55-63, 2003

[GiVe] J. Ginibre, G. Velo, On a class of nonlinear Schrödinger equations with nonlocal interaction, Math. Z. 170, 109-136, 1980

[He] K. Hepp, The classical limit for quantum mechanical correlation functions, Comm.Math.Phys 35, 265-267, 1974

[NS] H. Narnhofer, G. Sewell Vlasov hydrodynamics of a quantum mechanical model, Comm.Math.Phys, 79, 9-24, 1981

[Ro] D.Robert, Autour de l'approximation semiclassique, Birkhäuser Verlag, 1987

[Sp] H. Spohn, Kinetic equations from Hamiltonian dynamics: Markovian limits, Rev.Mod.Phys. 53, 569-615, 1980 